\title{
ASTROTURISMO COMO ALTERNATIVA ESTRATÉGICA DE DINAMIZACIÓN TERRITORIAL: EL CASO DE LA REGIÓN ESTRELLA DE CHILE
}

ASTROTOURISM AS STRATEGIC ALTERNATIVE OF TERRITORIAL DEVELOPMENT: THE CASE OF THE ESTRELLA REGION OF CHILE

\author{
ASTROTURISMO COMO ALTERNATIVA ESTRATÉGICA À \\ DINAMIZAÇÃO TERRITORIAL: O CASO DA REGIÃO ESTRELLA DO \\ CHILE
}

\section{Sebastián Araya-Pizarro ${ }^{1}$}

\section{Resumen}

Las condiciones privilegiadas de los cielos del norte de Chile han permitido que la región de Coquimbo se posicione como la capital mundial de la astronomía y forje una identidad local única: la de Región Estrella. No obstante, aún no ha logrado consolidar una oferta competitiva sistémica, que permita aprovechar la riqueza patrimonial que brinda el territorio. El 2 de julio de 2019, el país experimentó uno de los eventos astronómicos más importantes del orbe: el eclipse solar total, fenómeno que implicó el arribo de más de 300 mil pasajeros y brindó la oportunidad para explorar la complacencia de visitantes con su estancia. En este contexto, el presente estudio planteó por objetivo analizar el perfil y satisfacción del turista extranjero que participó del mega evento. Los datos se recopilaron a través de una encuesta aplicada a 228 turistas, y fueron, luego, analizados mediante una regresión logística binaria. Los resultados revelaron la existencia de diversos atributos del perfil del visitante y aspectos turísticos que influyen en su nivel de satisfacción (sexo, uso de e-commerce, oferta astronómica, alojamiento, señalización, limpieza, recursos financieros, entretenimiento e información turística). Se concluye sobre la necesidad de planificar estratégicamente el destino desde un enfoque integral sinérgico, que considere tanto los aspectos turísticos contextuales de la zona, como los atributos diferenciadores de la propuesta de valor ofrecida a los pasajeros. De este

Doi: https://doi.org/10.15359/eys.25-58.2 Recibido: 20-07-2020. Reenvíos: 17-09-2020. Aceptado: 22-09-2020. Publicado: 28-09-2020.

1 Académico, Departamento de Ciencias Económicas y Empresariales, Universidad de La Serena, Chile. Magíster en Liderazgo, Dirección Estratégica y Comunicación en las Organizaciones. Correo electrónico: saraya@userena.cl ORCID https://orcid.org/0000-0002-5857-8441

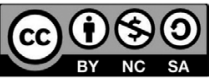


modo, se podría explotar el potencial que reviste el turismo astronómico como alternativa estratégica de dinamización territorial.

Palabras claves: Astroturista; satisfacción; turismo rural; eclipse solar; competitividad

\begin{abstract}
The privileged conditions of the skies in northern Chile have allowed the Coquimbo region to position itself as the world's capital of astronomy forging a unique local identity: that of the Estrella Region. However, no systemic competitive offer has been consolidated for the region to take advantage of the territory's patrimonial wealth. On July 2, 2019, the country experienced one of the most important astronomical events in the world, a total solar eclipse, a phenomenon that prompted the arrival of over 300 thousand visitors and provided the opportunity to explore their satisfaction with their visit. The objective of this study was to analyze the profile and satisfaction of foreign tourists who participated in the mega event. Data was collected through a survey applied to 228 tourists and was later analyzed using a binary logistic regression. Results revealed the existence of various attributes of the visitors' profile, as well as tourist aspects that influence their level of satisfaction (sex, use of e-commerce, astronomical offering, lodging, signage, cleanliness, financial resources, entertainment, and tourist information). It is concluded that there is a need to strategically plan the destination from a synergic holistic approach, considering both the contextual tourist aspects of the area and the differentiating attributes of the value proposition offered to passengers. In this way, the potential of astronomical tourism could be exploited as a strategic alternative for territorial revitalization.
\end{abstract}

Keywords: Astrotourist; satisfaction; rural tourism; solar eclipse; competitiveness

\title{
Resumo
}

As condições privilegiadas dos céus do norte do Chile permitiram que a Região de Coquimbo se posicionasse como a capital do mundo da astronomia e forjasse uma identidade local única: a da Região de Estrella. No entanto, ainda não conseguiram consolidar uma oferta competitiva sistêmica, que permita aproveitar a riqueza patrimonial fornecida pelo território. Em 2 de julho de 2019, o país viveu um dos eventos astronômicos mais importantes da orbe: o Eclipse Solar Total, fenômeno que envolveu a chegada de mais de 300 mil passageiros e proporcionou a oportunidade de explorar a complacência do visitante com sua estada. Nesse contexto, este estudo teve como objetivo analisar o perfil e a satisfação do turista estrangeiro que participou do megaevento. Os dados foram coletados por meio de uma pesquisa de levantamento com 228 turistas e analisados por meio de regressão logística binária. Os resultados revelaram a existência 
de diversos atributos do perfil do visitante e aspectos turísticos que influenciam seu nível de satisfação (sexo, uso de e-commerce, oferta astronômica, acomodação, sinalização, limpeza, recursos financeiros, entretenimento e informações turísticas). Conclui-se sobre a necessidade de planejar estrategicamente o destino sob uma abordagem holística sinérgica, que considera tanto os aspectos turísticos contextuais da área quanto os atributos diferenciadores da proposta de valor oferecida aos passageiros. Dessa forma, o potencial do turismo astronômico como alternativa estratégica ao dinamismo territorial poderia ser explorado.

Palavras-chave: Astroturista; satisfação; turismo rural; eclipse solar; competitividade

\section{Introducción}

La región de Coquimbo (Chile) es un destino turístico que se caracteriza por la diversidad de escenarios naturales que ofrece al visitante. Dispone de un litoral extenso con 56 kilómetros de playas y de un conjunto geográfico único de valles transversales que resaltan por sus condiciones climáticas, entorno paisajístico y cultural. Además, la región ostenta los cielos más diáfanos y limpios del planeta, lo que le permite sostener uno de sus atractivos principales: el astroturismo o turismo astronómico. Esta vertiente del turismo engloba al conjunto de actividades recreativas y educativas desarrolladas en torno al cosmos y los fenómenos astronómicos (Verde, 2016a), por lo que se conoce, igualmente, como el turismo de las estrellas (Prat, 2017) o ecoturismo celestial (Weaver, 2011).

Este tipo de turismo de intereses especiales (TIE), exclusivo y basado en la naturaleza, la cultura y su conservación (Espinosa et al., 2014; Fernández et al., 2015; Páramo \& Sánchez, 2018) ha posibilitado que Coquimbo desarrolle y fortalezca una identidad local: la de Región Estrella (González \& Morales, 2011). Esta fuente de diferenciación y valoración territorial se enmarca en una serie de hitos que han posicionado a la zona a la vanguardia del turismo astronómico mundial. Por ejemplo, en el 2013, obtuvo la primera certificación StarLight para el Parque Nacional Fray Jorge, inédita en Sudamérica y cuarta en el orbe. Y, en el 2015, el sitio astronómico del Observatorio AURA, en el Valle del Elqui, fue declarado, por la Asociación Internacional de Cielos Oscuros (IDA), como el primer Santuario Internacional de Cielos Oscuros del mundo.

No obstante las ventajas comparativas que presenta la región de Coquimbo para el desarrollo de la actividad turística, destacadas por la (Organisation for Economic Cooperation and Development, 2018), no ha logrado consolidar una oferta competitiva sistémica, que permita aprovechar la riqueza patrimonial que brinda el territorio. Así, en línea con lo que sucede en Chile, se registra una alta afluencia de turistas internacionales, pero que no se traduce en mayores ingresos. Por ejemplo, al comparar al país con otros destinos turísticos del hemisferio sur, se aprecia que percibe un gasto promedio por visita de USD 460.5, lo que representa menos del $50 \%$ de lo que reportan Brasil (USD 926) o Perú (USD 994) (Servicio Nacional de Turismo, 2017a; 2017b). Sumado a lo anterior, los 
resultados del índice chileno de competitividad turística regional (ICT), para Coquimbo, revelan importantes deficiencias que afectan la productividad del sector y limitan el desempeño competitivo de toda la industria (ICT, 2016).

El 2 de julio de 2019, la región experimentó el evento astronómico mundial más importante del año, el eclipse solar total, fenómeno cuya parte más visible (conocido como umbra) se produjo, justamente, en el Valle del Elqui. Por ello, existe coincidencia entre expertos y autoridades, respecto a que el evento marcó un punto de inflexión en el posicionamiento de Chile como destino astroturístico. En términos cuantitativos, el eclipse significó la visita de 307544 turistas a la región, reportó alrededor de USD 83 millones de ingresos, a través de hotelería, gastronomía y venta de souvenirs, y percibió un gasto promedio por visitante de USD 190 por día (Guerra, 2019).

De este modo, la naturaleza excepcional del mega evento significó para Chile, y en particular para la región de Coquimbo, una oportunidad única para explorar las características y expectativas de un turista diferente al que tradicionalmente visita la zona, más diverso en cuanto a su origen, con una mayor disposición a gastar y con una motivación distinta a la habitual, el turismo de sol y playa (Guerra, 2019).

En este contexto, el presente estudio planteó por objetivo analizar el perfil y el grado de satisfacción de turista extranjero que visitó el Eclipse Solar Total Chile 2019. Esto con la intención de aportar nueva evidencia empírica vinculada al desarrollo del astroturismo, una actividad que se perfila como una opción sostenible para el progreso socioeconómico de los territorios y un dinamizador de la economía de sus comunidades.

El artículo se divide en cinco secciones. Primero se describe el panorama del astroturismo de la región de Coquimbo. Segundo, se explica la metodología utilizada para analizar el perfil y los determinantes de la satisfacción del turista. Tercero, se sintetizan los principales resultados de la caracterización del visitante y del modelo estadístico aplicado (regresión logística binaria). Cuarto, se discuten los hallazgos del estudio para, finalmente, concluir sobre los aspectos que deben tomarse en cuenta para aprovechar el potencial que reviste el turismo astronómico.

\section{Astroturismo de la región de Coquimbo}

La Región de Coquimbo, se encuentra ubicada en Chile Central, abarca una superficie aproximada de $40580 \mathrm{~km}^{2}$, que se distribuye en tres provincias: Elqui, Limarí y Choapa. Limita al norte con la región de Atacama y al sur con la región de Valparaíso, al este con la República Argentina y al oeste con el océano Pacífico. La capital regional es la ciudad de La Serena. Esta zona posee diversos climas de transición entre desérticos y templados mediterráneos, y representa una de las dieciséis regiones que dividen administrativamente a Chile. La población es de 757586 habitantes, cifra que la ubica en el segundo lugar de los territorios que más han crecido durante la última década en el país (Instituto Nacional de Estadísticas, 2018). De acuerdo con la OECD, la superficie rural de la región es de $83 \%$ (12 de las 15 comunas) y el $26 \%$ de la población vive en zonas rurales (Oficina de Estudios y Políticas Agrarias, 2019). 
Las condiciones privilegiadas de los cielos del norte de Chile, junto a la importante capacidad astronómica instalada (que se proyecta se incrementará en la próxima década al $70 \%$ del total existente en el mundo) han permitido que el país se posicione como la capital mundial de la astronomía y la ventana hacia el universo (Ponce, 2018). En tal panorama, Coquimbo constituye la región más relevante de Chile, en términos de oferta y demanda del mercado del turismo astronómico, registra cerca del $47 \%$ de los oferentes astro turísticos (observatorios científicos, observatorios turísticos, alojamientos con oferta de astroturismo, tour operadores, planetarios y museos) y el $44 \%$ de las visitas del país (Véase la Figura 1). De allí que la región aspire a consolidarse como un referente internacional en la materia, como Canarias en España o la isla Stewart en Nueva Zelanda (Lacoste \& Navarrete, 2014).

Demanda astroturística de Chile 2014 (\%)

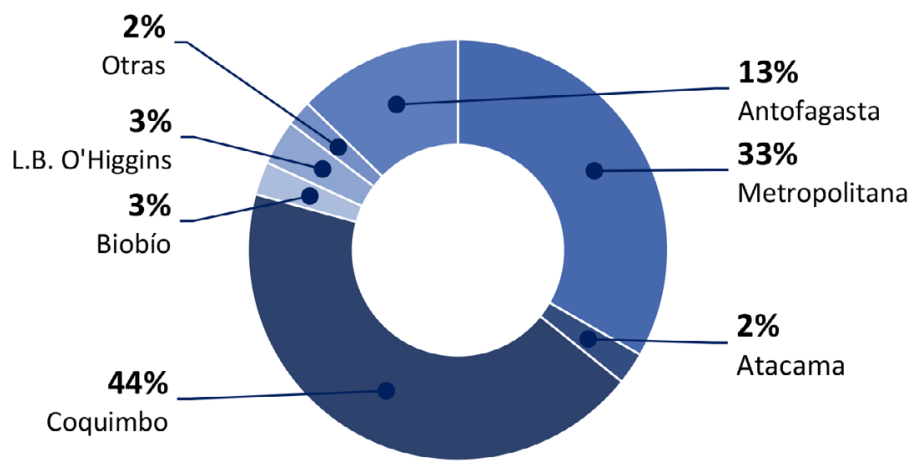

Oferta astroturística de Chile 2014 (\%)

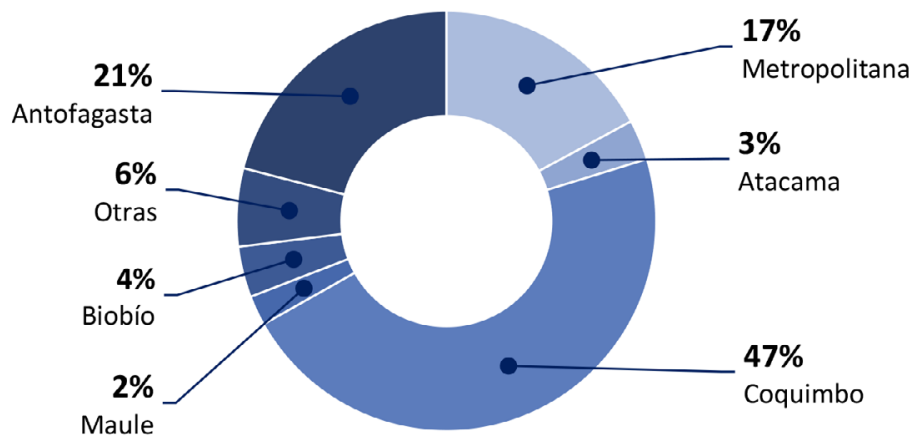

Figura 1. Participación regional en la demanda y oferta astroturística de Chile, 2014

Fuente: Elaboración propia con base en Verde (2016a, 2016b). 
La región de Coquimbo sustenta su desarrollo astroturístico en una serie de características y procesos propios, entre los que resaltan: (1) la calidad de sus cielos nocturnos, que brindan condiciones atmosféricas excepcionales para la contemplación astronómica (300 noches despejadas al año); (2) la baja contaminación lumínica, regulada por un marco normativo explícito (Decreto 43) que protege, de forma especial, la calidad ambiental de los cielos para el desarrollo de la investigación astronómica (Ministerio del Medio Ambiente, 2013); (3) el relieve propicio de altas cumbres (gran altitud); (4) las condiciones climáticas predominantes, tales como baja humedad relativa y bajas precipitaciones durante el año (clima árido) y; (5) la disponibilidad de infraestructura astronómica (observatorios científicos y turísticos) e infraestructura turística, como el alojamiento, transporte y servicios complementarios (Páramo \& Sánchez, 2018; Servicio Nacional de Turismo, 2018) .

Los oferentes astroturísticos regionales se distribuyen en las provincias de Elqui y Limarí. Entre estos se cuentan cuatro observatorios científicos internacionales, tres observatorios públicos y quince observatorios privados.

Cabe indicar, en cuanto al perfil de la oferta privada, que constituye un tejido productivo compuesto de pequeñas empresas, con recursos financieros escasos y bajos niveles de asociatividad, que ofrecen productos astroturísticos tradicionales como las visitas a observatorios, excursiones y observaciones a cielo abierto (nocturnas y diurnas). Datos trascendentes, si se considera que el turista moderno, y en especial el que disfruta del astroturismo, se caracteriza por su mayor nivel de conocimiento y exigencia (Araújo \& De Sevilha, 2017; Verde, 2016a).

\section{Metodología}

El estudio se desarrolló con un enfoque cuantitativo, de diseño no experimental, de tipo descriptivo y de corte transversal. Contó con la participación de 228 turistas extranjeros, cuyo número se computó en consideración de un nivel de confianza del 95 $\%$, un error muestral de $6.47 \%$, una heterogeneidad del $50 \%$ y un universo infinito. La recolección de datos se llevó a cabo mediante una encuesta estructurada presencial, con un muestreo intencional, aplicada posterior al evento, en los principales puntos de ingreso-salida a la región y lugares de observación del fenómeno. En específico, el cuestionario contó de 21 preguntas, organizadas en dos secciones: antecedentes sociodemográficos y comportamentales (sexo, edad, origen, ocupación, compañía, gasto, duración, planeación del viaje, experiencia previa, medio de información y uso de servicios online), y evaluación de la experiencia turística (servicios de entretención, señalización vial, disponibilidad de dinero, servicio de alojamiento, oferta astronómica, relación calidad-precio, limpieza del lugar, servicio de gastronomía, información turística, seguridad ciudadana, posicionamiento regional y hospitalidad de los residentes).

Los datos fueron examinados mediante técnicas de análisis estadístico descriptivo, correlacional e inferencial. Particularmente, se aplicaron pruebas de diferencias de medias (prueba T, prueba no paramétrica de Wilcoxon-Mann-Whitney y análisis de la varianza) y pruebas de independencia (chi-cuadrado) a un nivel de $5 \%$ de significancia,

Sebastián Araya-Pizarro 
que permitieron analizar la conformidad de los visitantes en función de diversas variables sociodemográficas y comportamentales.

Además, a fin de obtener un examen multivariado del nivel de satisfacción del turista (variable dependiente), se aplicó un análisis de regresión logística binario. Esta técnica estadística utiliza una variable de criterio dicotómica y una o más variables predictoras cualitativas, ordinales o cuantitativas. En este estudio, la conceptualización matemática para la satisfacción del visitante se definió de la siguiente forma:

$$
\log \left(\frac{p}{1-p}\right)=b_{0}+b_{1} x_{1}+b_{2} x_{2}+\cdots+b_{k} x_{k}
$$

En la ecuación, $p$ representa la probabilidad de que el turista extranjero esté satisfecho con su visita. De este modo, una respuesta positiva representa la satisfacción del turista (Sí $=1$ ) y una respuesta negativa su insatisfacción (No $=0$ ). Cabe mencionar que las variables explicativas del modelo derivaron primordialmente de dos secciones especiales del cuestionario (preguntas 17 y 20) que, diseñadas como una escala Likert de 5 puntos ( 1 = muy en desacuerdo/muy malo a $5=$ muy de acuerdo/muy bueno) recolectaron información respecto a diferentes dimensiones de satisfacción de aspectos turísticos definidos para el estudio, con base en el trabajo de Pasquotto et al. (2012): hospedaje, alimentación (restaurantes/cafés), informaciones turísticas, entretención, costos y elementos turísticos. La categorización de las variables de la escala Likert consistió en dicotomizarlas con base en dos niveles de satisfacción: satisfecho, cuando las respuestas fueron superiores a 3 (regular), e insatisfecho para los demás casos. También se adicionó un conjunto de variables sobre el perfil del turista (sexo, edad, ocupación, experiencia y uso de internet) y de tipo conductual (motivo, gasto de viaje, duración de la visita, experiencia previa, modo de viaje, tiempo de preparación y uso de medios de información online), que se estimaron podrían influir en el nivel de satisfacción.

Por lo demás, para determinar el poder predictivo y ajuste del modelo, se realizaron la prueba de Hosmeer \& Lemeshow y el $R$ cuadrado de Nagelkerke. La fiabilidad del instrumento se midió mediante el cómputo del coeficiente alfa de Cronbach, que mostró la pertinencia de la escala $(\alpha=.921)$. Por último, queda mencionar que todos los cálculos fueron realizados con el software estadístico IBM SPSS Statistics versión 24 para Windows.

\section{Resultados}

\subsection{Perfil del turista}

Los resultados revelan que los turistas extranjeros encuestados fueron hombres y mujeres (51\% y $49 \%$, respectivamente) con edades superiores a 30 años (80\%), provenientes de Estados Unidos (59 \%) y Europa (17\%). Son personas laboralmente activas (71 \%), que se desempeñan como empleados del sector privado (43\%) o autoempleados (empresarios o independientes, $20 \%$ ) (Véase la Tabla 1). 
Tabla 1

Características del perfil del turista

\begin{tabular}{llrr}
\hline Variable & Ítem & Frecuencia & Porcentaje \\
\hline Sexo & Hombre & 116 & 50.9 \\
& Mujer & 112 & 49.1 \\
\hline Edad & Menos de 30 & 45 & 19.7 \\
& $30-39$ años & 42 & 18.4 \\
& $40-49$ años & 29 & 12.7 \\
& 50-59 años & 36 & 15.8 \\
& 60-69 años & 47 & 20.6 \\
& 70 años o más & 29 & 12.7 \\
\hline Origen & Estados Unidos & 135 & 59.2 \\
& Inglaterra & 16 & 7.0 \\
& Alemania & 12 & 5.3 \\
& España & 10 & 4.4 \\
& Otro & 55 & 24.1 \\
\hline Ocupación & Autoempleado & 46 & 20.2 \\
& Empleado en el sector privado & 97 & 42.5 \\
& Empleado en el sector público & 18 & 7.9 \\
& Estudiante & 17 & 7.5 \\
& Ama de casa & 9 & 3.9 \\
& Jubilado & 41 & 18.0 \\
\hline
\end{tabular}

Fuente: Elaboración propia con base en resultados de la encuesta.

En relación con el comportamiento del turista, cabe indicar que estos viajaron acompañados (91 \%) y visitaron la región por primera vez (88 \%). En general, gastaron un monto superior a los CLP 400000 durante su estadía (67\%), con una permanencia promedio superior a dos noches (90\%). Además, planificaron su viaje con una anticipación superior a 3 meses ( $84 \%$ con más de un trimestre y un $50 \%$ con más de 1 año) y aun cuando se informó del destino turístico máxime por Internet (47\%), en su visita no contrataron mayormente servicios turísticos online (52\%). Sin embargo, quienes sí lo hicieron, usaron menos de dos servicios ( $82 \%)$, para alojamiento (68 menciones), transporte (64 menciones) y tour (39 menciones) (Véase la Tabla 2).

Tabla 2

Comportamiento de viaje del turista

\begin{tabular}{llrr}
\hline Variable & Ítem & Frecuencia & Porcentaje \\
\hline Con quien viaja & Solo & 21 & 9.2 \\
& Con familia & 88 & 38.6 \\
& Con amigos & 61 & 26.8 \\
& Con familia y amigos & 31 & 13.6 \\
& Otros & 27 & 11.8 \\
\hline
\end{tabular}




\begin{tabular}{|c|c|c|c|}
\hline Variable & Ítem & Frecuencia & Porcentaje \\
\hline \multirow[t]{5}{*}{ Nivel de gasto } & Menos de CLP 50.000 & 18 & 7.9 \\
\hline & CLP 50.001 - CLP 150.000 & 13 & 5.7 \\
\hline & CLP 150.001 - CLP 400.000 & 45 & 19.7 \\
\hline & CLP 400.001 - CLP 800.000 & 47 & 20.6 \\
\hline & Más de CLP 800.000 & 105 & 46.1 \\
\hline \multirow[t]{8}{*}{ Duración del viaje } & Se va el mismo día del evento & 6 & 2.6 \\
\hline & Una noche & 4 & 1.8 \\
\hline & Dos noches & 13 & 5.7 \\
\hline & Tres noches & 49 & 21.5 \\
\hline & De cuatro a siete noches & 84 & 36.8 \\
\hline & Más de siete noches & 72 & 31.6 \\
\hline & Menos de 1 semana & 6 & 2.6 \\
\hline & Entre 1 semana y 15 días & 7 & 3.1 \\
\hline \multirow{4}{*}{$\begin{array}{l}\text { Planeación del } \\
\text { viaje }\end{array}$} & Entre 15 días y 1 mes & 4 & 1.8 \\
\hline & Entre 1 mes y 3 meses & 20 & 8.8 \\
\hline & Entre 3 meses y 1 año & 78 & 34.2 \\
\hline & Más de 1 año & 113 & 49.6 \\
\hline \multirow[t]{2}{*}{ Primera visita } & Sí & 201 & 88.2 \\
\hline & No & 27 & 11.8 \\
\hline \multirow[t]{4}{*}{ Cómo se informó } & Internet & 106 & 46.5 \\
\hline & Agencia de viajes & 39 & 17.1 \\
\hline & $\begin{array}{l}\text { Recomendación de familiares/ } \\
\text { amigos }\end{array}$ & 48 & 21.1 \\
\hline & Otro & 35 & 15.4 \\
\hline \multirow{2}{*}{ Uso de e-commerce } & Sí & 109 & 47.8 \\
\hline & No & 119 & 52.2 \\
\hline
\end{tabular}

Fuente: Elaboración propia con base en resultados de la encuesta aplicada.

En cuanto al nivel de gasto, la aplicación de la prueba chi-cuadrado reveló asociaciones significativas con diferentes variables de caracterización del turista, tales como: la nacionalidad $(\chi 2=21.31, p<.01)$, el tipo de visita $(\chi 2=32.46, p<.01)$, la planificación del viaje $(\chi 2=27.76, p<.01)$, la duración de la estadía $(\chi 2=28.43, p<.01)$ y el número de acompañantes $(\chi 2=31.72, p<.01)$. En particular, se evidencia que quienes más gastaron fueron visitantes primerizos y de nacionalidad estadounidense. Además, se corroboró la relación directa del gasto con el tiempo de estadía, el número de acompañantes y el tiempo de preparación del viaje. Por otro lado, se detectó una asociación relevante entre la contratación de servicios online y la nacionalidad $(\chi 2=6.65, p<.05)$, que reveló que los estadounidenses contrataron menos servicios por esta línea ( $18 \%$ contrató más de uno) que los europeos (47 \% contrató más de 
uno). Sin embargo, no se hallaron diferencias significativas en la forma cómo los turistas se informan, es internet el medio preferido.

\subsection{Satisfacción del turista}

En general, la satisfacción del turista fue favorable. Resultó que el 87 \% se mostró satisfecho con su viaje, el 86 \% sintió que el viaje cumplió sus expectativas, el $83 \%$ recomendaría visitar el destino, el 82 \% valoró globalmente el servicio recibido como excelente y el $73 \%$ volvería a visitar la zona (Véase Figura 2).

\begin{tabular}{|c|c|c|}
\hline Volvería a visitar la Región de Coquimbo & $72.8 \%$ & $27.2 \%$ \\
\hline $\begin{array}{l}\text { Estoy satisfecho con mi visita a la Región de } \\
\text { Coquimbo }\end{array}$ & $87.3 \%$ & $12.7 \%$ \\
\hline $\begin{array}{c}\text { Recomendaría visitar la Región de } \\
\text { Coquimbo }\end{array}$ & $82.9 \%$ & $17.1 \%$ \\
\hline $\begin{array}{l}\text { La visita a la Región de Coquimbo cumplió } \\
\text { totalmente mis expectativas }\end{array}$ & $86.0 \%$ & $14.0 \%$ \\
\hline $\begin{array}{l}\text { Valoraría globalmente el servicio recibido } \\
\text { durante el evento como excelente }\end{array}$ & $81.6 \%$ & $18.4 \%$ \\
\hline
\end{tabular}

Figura 2. Indicadores de satisfacción del turista

Fuente: Elaboración propia con base en resultados de la encuesta aplicada.

El examen correlacional de las variables de satisfacción (coeficiente $r$ de Pearson) demostró un grado de asociación moderadamente significativo. Se advirtió que aquellos viajeros dispuestos a recomendar el destino son los que vieron cumplidas sus expectativas $(r=.654, p<.01)$, quedaron satisfechos con su viaje $(r=.721, p<.01), \mathrm{y}$ están dispuestos a repetir su visita $(r=.753, p<.01)$.

Cabe indicar que el contraste de medias no mostró diferencias relevantes $(p<.05)$ en el grado de satisfacción por sexo, edad u ocupación del visitante. No obstante, en relación con su origen, el análisis de la varianza mostró diferencias significativas con la disposición a recomendar el destino $(F=3.04, p<.05)$ e intención de repetir la visita ( $F=5.41, p<.01)$. En general, como ilustra la Figura 3, los turistas estadounidenses mostraron, en ambas dimensiones, una mayor valoración. 


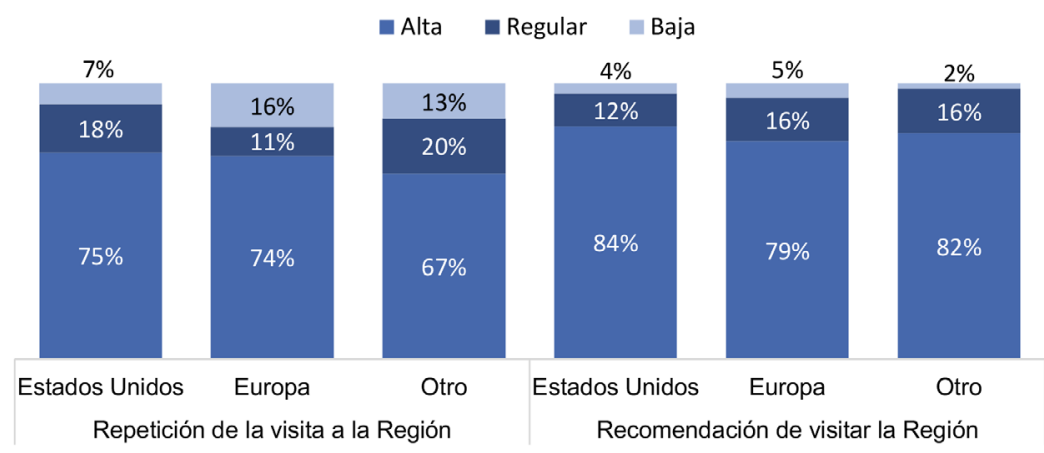

Figura 3. Nivel de valoración por dimensión de satisfacción según origen del turista.

Fuente: Elaboración propia con base en resultados de la encuesta aplicada.

Por otro lado, el análisis de regresión logística binaria, se realizó introduciendo, gradualmente, las tres categorías de variables del estudio (demográficas, comportamentales y atributos de satisfacción). Al respecto, se obtuvieron tres modelos de regresión, cuyos resultados se muestran en la Tabla 3.

Tabla 3

Regresión logística binaria con las variables del modelo

\begin{tabular}{|c|c|c|c|c|c|c|}
\hline \multirow[b]{2}{*}{ Variable } & \multicolumn{2}{|c|}{ Modelo 1} & \multicolumn{2}{|c|}{ Modelo 2} & \multicolumn{2}{|c|}{ Modelo 3} \\
\hline & OR & 95\% C.I. & OR & 95\% C.I. & OR & 95\% C.I. \\
\hline Sexo (masculino) & $1.81 *$ & {$[0.89,3.68]$} & $2.12 * *$ & {$[1.00,4.50]$} & $2.99 * *$ & {$[1.09,8.15]$} \\
\hline Edad (>=40 años) & 1.74 & {$[0.84,3.59]$} & 1.30 & {$[0.57,2.98]$} & 1.63 & {$[0.56,4.68]$} \\
\hline Ocupación (dependiente) & $3.06 * * *$ & {$[1.39,6.69]$} & $3.35^{* * *}$ & {$[1.48,7.59]$} & 2.13 & {$[0.74,6.14]$} \\
\hline Nacionalidad (USA) & $2.03 * *$ & {$[1.01,4.08]$} & 1.80 & {$[0.86,3.76]$} & 1.56 & {$[0.59,4.16]$} \\
\hline Nivel de gasto (bajo) & & & 1.42 & {$[0.44,4.55]$} & 0.54 & {$[0.12,2.34]$} \\
\hline Acompañado & & & 2.48 & {$[0.71,8.64]$} & 4.16 & {$[0.73,23.8]$} \\
\hline Duración visita (<4 noches) & & & 1.11 & {$[0.49,2.53]$} & 1.58 & {$[0.51,4.89]$} \\
\hline $\begin{array}{l}\text { Planeación visita (>=1 } \\
\text { mes) }\end{array}$ & & & 1.97 & {$[0.46,8.41]$} & 0.82 & {$[0.12,5.75]$} \\
\hline Primera visita & & & 0.92 & {$[0.28,3.01]$} & 0.25 & {$[0.04,1.51]$} \\
\hline Se informa por Internet & & & 0.65 & {$[0.28,1.53]$} & & \\
\hline Usa e-commerce & & & 0.93 & {$[0.42,2.04]$} & $0.24 * *$ & {$[0.08,0.73]$} \\
\hline Servicio de alojamiento & & & & & $4.22 * * *$ & {$[1.49,11.9]$} \\
\hline Servicio de gastronomía & & & & & 0.83 & {$[0,28,2.48]$} \\
\hline Calidad-precio de la zona & & & & & 2.20 & {$[0.78,6.15]$} \\
\hline Limpieza del lugar & & & & & $5.35^{* * *}$ & {$[1.74,16.5]$} \\
\hline Información turística & & & & & $4.14 * * *$ & {$[1.47,11.6]$} \\
\hline
\end{tabular}




\begin{tabular}{|c|c|c|c|c|c|c|}
\hline \multirow[b]{2}{*}{ Variable } & \multicolumn{2}{|c|}{ Modelo 1} & \multicolumn{2}{|c|}{ Modelo 2} & \multicolumn{2}{|c|}{ Modelo 3} \\
\hline & OR & 95\% C.I. & OR & 95\% C.I. & OR & 95\% C.I. \\
\hline Señalización vial & & & & & $2.96 * *$ & {$[1.03,8.51]$} \\
\hline Oferta astronómica & & & & & $2.38^{*}$ & {$[0.88,6.44]$} \\
\hline Disponibilidad de dinero & & & & & $3.69 * *$ & {$[1.25,10.9]$} \\
\hline $\begin{array}{l}\text { Hospitalidad de los } \\
\text { residentes }\end{array}$ & & & & & 1.72 & {$[0.42,6.97]$} \\
\hline Servicios de entretención & & & & & $0.35^{*}$ & {$[0.11,1.11]$} \\
\hline Seguridad ciudadana & & & & & 1.17 & {$[0.35,3.89]$} \\
\hline Posicionamiento regional & & & & & 2.13 & {$[0.71,6.34]$} \\
\hline Constante & 1.08 & & 0.21 & & $0.02 * * *$ & \\
\hline $\begin{array}{l}\text { Prueba de Hosmery } \\
\text { Lemeshow }\end{array}$ & .90 & & .84 & & .35 & \\
\hline R cuadrado de Cox y Snell & .06 & & .08 & & .33 & \\
\hline R cuadrado de Nagelkerke & .10 & & .13 & & .53 & \\
\hline AUC & .68 & & .70 & & .90 & \\
\hline
\end{tabular}

Notas: $\mathrm{OR}=$ Odds ratio. AUC = Área bajo la curva. ${ }^{*} p<.10 ;{ }^{* *} p<.05 ;{ }^{* * *} p<.01$. Variable dependiente: variable dicotómica que evalúa que el turista esté satisfecho con su visita o no. Elaboración propia con base en la encuesta aplicada.

En la ecuación final (modelo 3), se revisó la influencia de 23 variables explicativas sobre la satisfacción del turista, de las cuales nueve resultaron ser significativas. Los hallazgos muestran que los factores con mayor poder de predicción fueron: la limpieza de la zona, el servicio de alojamiento, la información turística provista al visitante, la disponibilidad de dinero, la señalización, la oferta astronómica y los servicios de entretención. Además, los resultados permitieron constatar que existe más probabilidad de satisfacción en turistas de sexo masculino y que utilizan el e-commerce (contratación de servicios online).

Por otra parte, la aplicación de la prueba de Hosmer y Lemeshow corroboró el ajuste adecuado del modelo ( $p>.05$ ). Y el coeficiente $R$ cuadrado de Nagelkerke evidenció que las variables independientes de la ecuación de regresión logística explican aproximadamente el 53 \% de la satisfacción del turista (poder explicativo moderado), con una buena capacidad de discriminación $(A \cup C=.90)$.

Por último, la revisión específica de las respuestas, según atributo de satisfacción, permitió identificar tres categorías de evaluación: nivel alto (mayor a $70 \%$ ), nivel medio (entre $50 \%$ y $70 \%$ ) y nivel bajo (menor a $50 \%$ ). Los elementos mejor evaluados fueron: la información turística y la limpieza del lugar. Luego les siguen la oferta astronómica, el servicio de alojamiento y la disponibilidad de dinero. Y, finalmente, los aspectos deficitarios fueron la señalización vial y el servicio de entretención (Véase la Figura 4). 


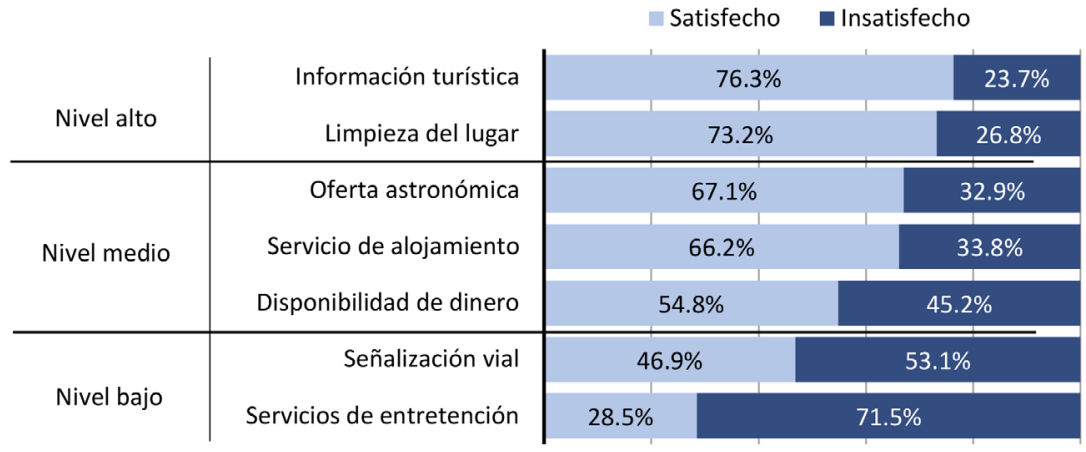

Figura 4. Niveles de satisfacción según criterio de evaluación

Fuente: Elaboración propia con base en resultados de la encuesta aplicada.

\section{Discusión}

De acuerdo con los resultados referidos al perfil del turista, los hallazgos mostraron correspondencia con lo expuesto por Verde (2016a), quien caracterizó al segmento como visitantes, principalmente, estadounidenses, de entre 20 y 60 años, que cuentan con un alto nivel cultural, que planifican sus viajes con antelación y se informan sobre la oferta astroturística por internet (foros y revistas especializadas). Este último punto permite confirmar la relevancia creciente de esta red como fuente de información y mecanismo transaccional (e-commerce).

Lo anterior demuestra el potencial que presenta internet como herramienta para la proyección de la imagen turística de los destinos y la promoción de los diversos atractivos de la zona (Hidalgo-Alcázar et al., 2015; López et al., 2017). Asimismo, devela el desafío, para todos los actores locales que integran la cadena de servicio, de instaurar y desarrollar capacidades digitales (creación de contenido multimedia y uso de herramientas tecnológicas innovadoras) acordes con los requerimientos y estándares de un visitante exigente, inserto en la era del e-business. Este aspecto es esencial, si se consideran los rasgos propios deficitarios de las regiones rurales. Por un lado, su evolución tecnológica limitada y escasa, tanto a nivel de la infraestructura disponible como de capacidades empresariales. Y por otro, el desarrollo organizacional económico incipiente de sus oferentes, que se enmarca en un sistema de baja densidad económica y espacial (Verde, 2016b). De allí que se argumente la necesidad de idear una economía cooperativa y articulada geográficamente, donde las unidades empresariales urbanas y rurales de la región puedan aprovechar los recursos naturales y culturales propios del territorio, desde un enfoque de colaboración sinérgica.

Los resultados del examen correlacional confirmaron lo expuesto por otros autores, en cuanto al rol esencial que desempeña el cumplimiento de las expectativas del pasajero para incrementar la intención de que estos repitan su visita y atraigan nuevos turistas por 
sus recomendaciones (Campón-Cerro, et al., 2017; Oliveira, 2011; Weaver et al., 2007). Además, se desprende la oportunidad para explorar los satisfactores que ocasionan las diferencias percibidas en el estudio, en cuanto a la conformidad según sexo.

Del análisis multivariante se revela, primero, que los turistas destacan como fortalezas, en primer lugar, la información brindada al visitante y el cuidado-limpieza del lugar. Segundo, se identificaron tres factores, cuyo fortalecimiento conllevaría a mejorar la experiencia: la diversidad de la oferta astroturística, la cantidad de cajeros/casas de cambio y la calidad del servicio de alojamiento. Y, tercero, se halló que hay dos debilidades por mejorar: la señalización disponible y la oferta complementaria de ocio. Tales hallazgos resaltan la importancia de preocuparse por aquellos factores exógenos que resultan determinantes para la competitividad del sector, pero que requieren del apoyo de las autoridades regionales y el trabajo mancomunado de los agentes privados. Solo de esta manera, podría establecerse una infraestructura turística apropiada para ofrecer un servicio de excelencia. Sobre todo, en lo vinculado a tópicos sindicados como prioritarios en la literatura del turismo, como la limpieza (Benseny, 2006), la oferta de servicios de ocio (Alegre \& Garau, 2009) y el alojamiento (Fuentes, et al., 2016).

También, sobresale el rol de la información provista al turista, pues junto con atender las exigencias singulares del turismo astronómico, son vitales para la promoción de las diferentes actividades de entretenimiento de la región. Por ello, la capacitación de los responsables de proveer la información es clave. Zárraga et al. (2010), por ejemplo, resaltan la influencia de los guías turísticos en la experiencia que los visitantes tienen de un destino, pues son, en gran parte, responsables de la imagen que un turista se lleva a su país.

En relación con la valoración de la oferta astronómica, los resultados advierten la pertinencia de que los responsables de promover el turismo astronómico se preocupen por configurar una oferta integral y diversa, que potencie, junto a los elementos naturales del territorio, otros aspectos turísticos, tales como: la infraestructura del lugar, las facilidades recreativas, la atención al visitante, y el ofrecimiento de actividades innovadoras y complementarias al astroturismo. En atención a esto, Alegre \& Garau (2009) posicionan la variedad de la oferta de productos turísticos, como el factor de mayor poder de predicción de repetición de visita de un destino.

Por esto, para la diversificación del astroturismo, es necesario abarcar un marco amplio de actividades turísticas que, sustentadas en la cultura del cielo y las estrellas, logren combinarse para generar un producto turístico potenciado en las comunidades donde se implanta. Entre tales actividades, se pueden contar: la restauración (gastronomía), la fotografía (astrofotografía), la arqueología (arqueoastronomía), la música (astromúsica), la ludificación (starparties), la artesanía (mística del cielo), el termalismo (a la luz de las estrellas), el senderismo y las cabalgatas para la contemplación nocturna, entre otras.

Al respecto, existen estudios que destacan la importancia de desarrollar una propuesta memorable que integre la visión del destino y del turista (Carballo et al., 2015). En este sentido, Pine II \& Gilmore (1998) identificaron cinco principios claves para diseñar 
experiencias memorables, los cuales son: establecer para la experiencia un tema claro y convincente, armonizarla con señales positivas, eliminar las señales negativas, mezclarla con recuerdos atractivos e involucrar en su configuración los cinco sentidos. Por ello, la tematización de los espacios en torno a la astronomía significaría una estrategia válida para optimizar el valor de la experiencia del turista, empero, debe lograr integrarse con la identidad e historia particular de las comunidades locales. Por ejemplo, en el aspecto cultural, con la poetisa regional Gabriela Mistral o, en el aspecto gastronómico, con productos típicos como el pisco, el que cuenta con denominación de origen para la región de Coquimbo. Este patrimonio natural y cultural del territorio se proyecta como un activo generador del progreso local y fuente de diferenciación regional.

\section{Conclusiones}

Los resultados de la investigación poseen distintas implicancias tanto para los responsables de definir las políticas públicas en materia turística en la región, como para los empresarios astroturísticos de las comunidades involucradas. Por una parte, denota la existencia de ciertas características del viajero que son un referente importante para la planeación estratégica del desarrollo del astroturismo de las regiones rurales. Y, por otra, establece el efecto predictivo de diversos factores que influyen en la satisfacción y repetición de la visita del turista.

Primero, en atención el perfil del visitante, se advierte la necesidad de establecer una oferta astronómica integral y diversa, que considere, junto a los atractivos naturales del territorio, la infraestructura de apoyo (física, comercial, recursos humanos y financieros), los servicios, equipamientos locales y la tematización del establecimiento astroturístico; lo que influiría de manera positiva en la percepción de la experiencia turística. Además, dada la relevancia de internet como medio informativo y transaccional, la aplicación de técnicas de marketing digital se vislumbra como una opción valiosa que permitiría acortar las distancias de las comunidades rurales, geográficamente distantes, y acercar la oferta astronómica e identidad local a los clientes extranjeros.

Por su parte, los factores con mayor poder de predicción de la satisfacción del turista (sexo, uso de e-commerce, limpieza, información turística, señalización, entretención, disponibilidad de dinero, alojamiento y oferta astroturística) revelan la significancia de planificar estratégicamente el destino, considerando tanto los aspectos turísticos contextuales del territorio, como los atributos diferenciadores de la propuesta de valor ofrecida a los visitantes. En este último punto, es importante estudiar la articulación de un programa turístico integral que, mediante la combinación de la astronomía, gastronomía, cultura, entretenimiento, etc., permita prolongar la estadía y cantidad de visitas de los diferentes segmentos potenciales. Esto podría impulsar la competitividad y el dinamismo del sector.

Para futuras investigaciones, se sugiere replicar este trabajo en los diferentes destinos astroturísticos de las comunidades en sus contextos particulares (social, económico y cultural) a fin de verificar la consistencia de los hallazgos. Así también, se recomienda realizar un estudio de carácter longitudinal que posibilite revisar la evolución de los

Sebastián Araya-Pizarro 
factores subyacentes de satisfacción, y comprender cómo estos afectan la repetición de la visita. Por último, se sugiere complementar el análisis del perfil del astroturista, adhiriendo un estudio explicativo sobre las motivaciones, las disconformidades y los aspectos que influyen en la formación de sus expectativas. De este modo, podrán desarrollarse productos más diferenciados y adecuados a las preferencias del turista, que conlleven al aprovechamiento del potencial económico local.

\section{Referencias}

Alegre, J., \& Garau, J. (2009). The factor structure of tourist satisfaction at sun and sand destinations. Journal of Travel Research, 50(1), 78-86. Doi: https://doi.org/10.1177/0047287509349270

Araújo, G., \& De Sevilha, M. (2017). Los viajeros y sus motivaciones. Un estudio exploratorio sobre quienes aman viajar. Estudios y Perspectivas en Turismo, 26(1), 62-85. Recuperado de https://www.redalyc.org/pdf/1807/180749182004.pdf

Benseny, G. (2006). El espacio turístico litoral. Aportes y Transferencias, 10(2), 102-122. Recuperado de https://www.redalyc.org/pdf/276/27610208.pdf

Campón-Cerro, A., Hernández-Mogollón, J., \& Alves, H. (2017). Sustainable improvement of competitiveness in rural tourism destinations: The quest for tourist loyalty in Spain. Journal of Destination Marketing and Management, 6(3), 252-266. Doi: https://doi.org/10.1016/j. jdmm.2016.04.005

Carballo, R., Moreno-Gil, S., León, C., \& Brent, J. R. (2015). La creación y promoción de experiencias en un destino turístico. Un análisis de la investigación y necesidades de actuación. Cuadernos de Turismo, (35), 71-94. Doi: https://doi.org/10.6018/turismo.35.221511

Espinosa, A., Llancaman, L., \& Sandoval, H. (2014). Turismo de intereses especiales y parques nacionales. Compatibilidad entre turismo de intereses especiales y gestión de parques nacionales. Estudios y Perspectivas del Turismo, 23(1), 115-130. Recuperado de http://www. redalyc.org/articulo.oa?id=180729920007

Fernández, C., Cea, J., Santander, P., \& Melo, R. (2015). Turismo de intereses especiales: Investigación de mercados sobre las motivaciones desde la perspectiva del cliente. Revista Internacional Administracion y Finanzas, 8(1), 5-9. Recuperado de ftp://ftp.repec.org/opt/ReDIF/ RePEc/ibf/riafin/riaf-v8n1-2015/RIAF-V8N1-2015-4.pdf

Fuentes, M., Hernández, E., \& Morini, S. (2016). Q de calidad y satisfacción del turista en el sector hotelero español. Cuadernos de Turismo, (37), 203-226. Doi: https://doi.org/10.6018/ turismo.37.256211

González, F., \& Morales, R. (2011). Análisis del turismo de la región de Coquimbo. Revista Interamericana de Ambiente y Turismo - RIAT, 7(2), 80-88. Recuperado de https://riat.utalca.cl/ index.php/test/article/view/58

Guerra, I. (2019, julio 4). Efecto eclipse: Región de Coquimbo registró ingresos por \$56 mil millones por el evento astronómico. El Mercurio. Recuperado de https://www.emol.com/noticias/ Economia/2019/07/04/953562/Efecto-eclipse-Region-de-Coquimbo-consiguio-ingresos-por-56-mil-millones-por-el-fenomeno-astronomico.html

Hidalgo-Alcázar, C., Sicilia, M., \& Ruiz De Maya, S. (2015). La imagen de un producto turístico rural a través del acceso al contenido generado por otros usuarios en internet: Diferencias por género. Journal of Technology Management and Innovation, 10(3), 75-84. Doi: https:// doi.org/10.4067/S0718-27242015000300009

Índice Chileno de Competitividad Turística Regional. (2016). ICT Chile; resultados por región, Coquimbo. Recuperado de http://ictchile.unab.cl/region.html 
Instituto Nacional de Estadísticas. (2018). Sintesis de resultados Censo 2017. Recuperado de https://www.censo2017.cl/descargas/home/sintesis-de-resultados-censo2017.pdf

Lacoste, P., \& Navarrete, S. (2014). Alternativas no tradicionales de desarrollo rural: la Ruta del Pisco como recurso turístico (valle de Elqui, Chile). Idesia (Arica), 32(4), 5-14. Doi: https:// doi.org/10.4067/S0718-34292014000400002

López, A. L., Verdesoto, E., \& López, A. (2017). Turismo 2.0 como herramienta para promocionar los atractivos culturales de Guayaquil. INNOVA Research Journal, 2(6), 154-163. Doi: https://doi.org/10.33890/innova.v2.n6.2017.299

Ministerio del Medio Ambiente. (2013). Decreto 43. Establece norma de emisión para la regulación de la contaminación lumínica, elaborada a partir de la revisión del Decreto $n .^{\circ} 686$, de 1998, del Ministerio de Economía, Fomento y Reconstrucción. Recuperado de https:// www.leychile.cl/N?i=1050704\& $\mathrm{f}=2014-05-03 \& \mathrm{p}=$

Oficina de Estudios y Políticas Agrarias. (2019). Región de Coquimbo. Informativo regional. Recuperado de https://www.odepa.gob.cl/wp-content/uploads/2019/04/Region-de-Coquimbo.pdf

Organisation for Economic Co-operation and Development. (2018). OECD Tourism trends and policies, 2018. Doi: https://doi.org/10.1787/20767773

Oliveira, B. (2011). Determinantes de la satisfacción del turista. Un estudio en la ciudad de Guarujá-Brasil. Estudios y Perspectivas en Turismo, 20(1), 229-242. Recuperado de https://www. redalyc.org/pdf/1807/180717677013.pdf

Páramo, J. D. D., \& Sánchez, Á. (2018). Estructura territorial del turismo astronómico en la región de Coquimbo, Chile. Revista Geográfica de América Central, 3(61E), 181-206. Doi: https:// doi.org/10.15359/rgac.61-3.10

Pasquotto, M., Monfort, M., \& De Oliveira, D. (2012). Elementos determinantes de la satisfacción del consumidor de servicios turísticos. Estudios y Perspectivas en Turismo, 21(5), 12441261. Recuperado de http://www.estudiosenturismo.com.ar/MT-MD-v21-5.htm

Pine II, J., \& Gilmore, J. (1998). Welcome to the Experience Economy. Harvard Business Review, (November 1998), 97-105. Recuperado de https://hbr.org/1998/07/ welcome-to-the-experience-economy

Ponce, M. (2018). Cielos de Chile: Desde la Tierra al Universo. Recuperado de https://mma.gob.cl/ wp-content/uploads/2018/06/Cielos_2018_Chilean_Skies.pdf

Prat, J. M. (2017). El caso de la Comarca de la Sierra Sur de Jaén. En G. Cànoves, A. Blanco-Romero, J. M. Prat, \& M. Villarino (Eds.), Turismo de interior en España. Productos y dinámicas territoriales, (Primera ed, 187-195). España: Publicacions de la Universitat de València. Recuperado de https://dialnet.unirioja.es/servlet/libro?codigo=702665

Servicio Nacional de Turismo. (2017a). Anuario de turismo 2016. Recuperado de https://www. sernatur.cl/wp-content/uploads/2018/09/20170731-ANUARIO-TURISMO-2016-julio.pdf

Servicio Nacional de Turismo. (2017b). Manual de diseño experiencias turísticas. Recuperado de https://www.sernatur.cl/wp-content/uploads/2018/11/MDD-Manual-de-Experiencias-Turísticas-1.pdf

Servicio Nacional de Turismo. (2018). Astroturismo. La Serena, Chile: Servicio Nacional de Turismo Región de Coquimbo. Recuperado de http://turismoregiondecoquimbo.cl/wp-content/ uploads/2019/05/Astroturismo2019.pdf

Verde. (2016a). Estudio sobre la demanda astroturística en Chile. Recuperado de https://www. academia.edu/35903268/estudio_sobre_la_demanda_astroturística_en_chile 
Verde. (2016b). Estudio sobre la oferta astroturística en Chile. Recuperado de https://www.academia.edu/35903206/estudio_sobre_oferta_de_astroturismo_en_chile

Weaver, D. (2011). Celestial ecotourism: New horizons in nature-based tourism. Journal of Ecotourism, 10(1), 38-45. Doi: https://doi.org/10.1080/14724040903576116

Weaver, P., Weber, K., \& Mccleary, K. (2007). Destination evaluation: The role of previous travel experience and trip characteristics. Journal of Travel Research, 45(3), 333-344. doi: https:// doi.org/10.1177/0047287506292702

Zárraga, L., Corona, E., \& Molina, V. (2010). Las competencias de los guías turistas como ventaja competitiva en el sector turista: Caso Cancún Quintana Roo. Revista Internacional Administración \& Finanzas, 3(3), 11-25. Recuperado de https://papers.ssrn.com/sol3/papers. cfm?abstract_id=1881174 